\title{
COMPREENSÃO DE GESTANTES E PUÉRPERAS SOBRE O PROGRAMA DE HUMANIZAÇÃO NO PRÉ-NATAL E NASCIMENTO EM UM MUNICÍPIO DO MARANHÃO, BRASIL
}

\begin{abstract}
Monyka Brito Lima dos Santos*1; Nivya Carla de Oliveira Pereira Rolim²; Ana Helia de Lima Sardinha3; Elza Lima da Silva4; Raylena Martins da Costa5; Jordeilson Luis Araújo Silva6; Jesica Cristina Lira dos Santos $^{7}$; Cristiane Michele Sampaio Cutrim ${ }^{8}$; Vera Alice Oliveira Viana ${ }^{9}$; Jucelene Soares de Macêdo ${ }^{10}$; Luciana Moraes de Oliveira ${ }^{11}$; Milka Borges da Silva ${ }^{12}$; Suzane Laura Silva de Carvalho' ${ }^{13}$; Vanessa Nunes Vasconcelos ${ }^{14}$; Ricardo Clayton Silva Jansen ${ }^{15}$; Gabriela Oliveira Parentes da Costa ${ }^{16}$; Aclenia Maria Nascimento Ribeiro' ${ }^{17}$; Marília Rosendo Rodrigues Soares ${ }^{18}$ and Ivo Ferreira de Santana ${ }^{19}$
\end{abstract}

${ }^{1}$ Enfermeira, Especialista em Ginecologia e Obstetrícia pela Faculdade Unyleya; ${ }^{2}$ Enfermeira, Mestranda em Enfermagem pela Universidade Federal do Maranhão; ${ }^{3}$ Enfermeira, Doutora em Ciências Pedagógicas pela Universidade Federal de Santa Catarina; ${ }^{4}$ Enfermeira, Doutora em Ciências: Fisiopatologia Clínica e Experimental pela Faculdade de Ciências Médicas pela Universidade Estadual do Rio de Janeiro; ${ }^{5}$ Mestranda em Enfermagem pela Universidade Federal do Maranhão; ${ }^{6}$ Enfermeiro, Graduado pela Faculdade IESM; ${ }^{7}$ Enfermeira, Residente em Enfermagem Obstétrica pela Universidade Estadual do Maranhão; ${ }^{8}$ Enfermeira, Mestranda em Saúde e Ambiente pela Universidade Federal do Maranhão; ${ }^{9}$ Enfermeira, Mestranda em Saúde e Comunidade pela Universidade Federal do Piauí; 10Enfermeira, Pós-graduanda em Enfermagem do Trabalho pelo Instituto de Ciências, Educação e Tecnologia; 11Enfermeira, Especialistaem Terapia Intensiva pela Uninovafapi; ${ }^{12}$ Enfermeira, Residente em Enfermagem Obstétrica pela Universidade Estadual do Maranhão; ${ }^{13}$ Enfermeira, Especialista em Saúde da Família e Comunidade pela Unasus; ${ }^{14}$ Enfermeira, Graduada pela Universidade Federal do Maranhão; ${ }^{15}$ Enfermeiro, Mestre em Biodiversidade, Ambiente e Saúde pela Universidade Estadual do Maranhão; 16Enfermeira, Mestranda em Enfermagem Universidade Federal do Piauí; 17Enfermeira, Residente em Alta Complexidade pela Universidade Federal do Piauí; ${ }^{18}$ Enfermeira Especialista multiprofissional em Nefrologia pela Uninter; ${ }^{19}$ Enfermeiro, Especialista em Estratégia Saúde da Família e Gestão em Saúde pela Universidade Federal do Piaú.

\section{ARTICLE INFO}

\section{Article History:}

Received $15^{\text {th }}$ December, 2020

Received in revised form

$20^{\text {th }}$ December, 2020

Accepted $19^{\text {th }}$ January, 2021

Published online $28^{\text {th }}$ February, 2021

\section{Key Words:}

CuidadoPré-natal, Gravidez,

Parto Humanizado, Período Pós-Parto,

Direito à Saúde.

\begin{abstract}
Objetivo: Investigar a compreensão de gestantes e puérperas sobre o Programa de Humanização no Pré-Natal e Nascimento demonstrando que o nível de entendimento pode ser uma premissa no cumprimento de seus direitos a assistência humanizada. Métodos: Pesquisa exploratóriadescritiva, com abordagem qualitativa, realizada em uma maternidade pública. Amostra composta por 15 gestantes e puérperas, dados obtidos a partir de entrevistas semiestruturadas com perguntas abertas. Resultados: Dentre as quinze gestantes e puérperas, apenas uma afirmou conhecer o PHPN, evidenciando o déficit na orientação em saúde das gestantes durante a execução do pré-natal da Unidade Básica de Saúde. Os discursos da amostra pesquisada demonstram que os profissionais de enfermagem que realizam os pré-natais estão alheios as gestantes, não cumprindo adequadamente com seu dever de orientar e informar sobre seus direitos frente ao programa, sanando duvidas que podem lhes assegurar uma assistência humanizada e acolhedora. Conclusão: É fundamental a colaboração dos enfermeiros na promoção da saúde. Conhecer o Programa de Humanização no Pré-Natal e Nascimento traz autonomia para a gestante e participação efetiva no seu processo gestacional e parturitivo, uma vez que, compreender sobre o programa pode ser uma premissa para exigir o cumprimento de seus direitos a assistência humanizada.
\end{abstract}

*Corresponding author:

Monyka Brito Lima dos Santos,

Copyright (C) 2020, Monyka Brito Lima dos Santos et al. This is an open access article distributed under the Creative Commons Attribution License, which permits unrestricted use, distribution, and reproduction in any medium, provided the original work is properly cited.

Citation: Monyka Brito Lima dos Santos; Nivya Carla de Oliveira Pereira Rolim; Ana Helia de Lima Sardinha; Elza Lima da Silva; Raylena Martins da Costa; Jordeilson Luís Araújo Silva, et al., 2021. "Compreensão de gestantes e puérperas sobre o programa de humanização no pré-natal e nascimento em um município do maranhão, Brasil”, International Journal of Development Research, 11, (02), 44692-44695.

\section{INTRODUCTION}

A Política Nacionalde Saúde da Mulher era muito restrita no que diz respeito agravidez e parto, neste contexto, foi regulamentado pela portaria GM/MS no 569 no ano de 2000, o Programa de Humanização no Pré-Natal e Nascimentono no âmbito do SUS, com o intuito de assegurar a melhoria do acesso a saúde, a cobertura e qualidade do acompanhamento pré-natal e assistência ao parto e puerpério às gestantes e ao recém-nascido (Brasil, 2002). Tendo em vista a melhoria da assistência ao parto, o Ministério da Saúde, a partir da Lei $n^{\circ} 11.108 / 2005$ garantiu às parturientes o direito à presença do acompanhante durante o trabalho de parto, parto e pós-parto imediato 
no âmbito do SUS, tornando o parto mais humanização. Lançou ainda a Lei $n^{0} 11.634 / 2007$ que dispunha sobre o direito da gestante ao conhecimento e à vinculação à maternidade onde receberia a assistência no âmbito do SUS (Diniz et al., 2014; Brasil, 2012). Embora tenham sido criadas leis e portarias que fortalecessem a assistência integral à saúde da mulher na gravidez, parto e puerpério, o Brasil ainda apresenta falhas na prática de humanizar a assistência em maternidades públicas, apesar do desenvolvimento teórico-prático no campo da obstetrícia, o trabalho de parto ainda está envolto por intervenções desnecessárias, exagero de cesarianas, isolamento de mulheres grávidas de familiares e desrespeito a sua liberdade de escolha, com isso, os indicadores de saúde apontam que no Brasil o cuidado obstétrico requer melhorias na humanização (Dodou; Rodrigues; Oriá, 2017). A humanização é um método necessários para remodelar a assistência desumana prestada em maternidades públicas, almejando mudanças no exercício profissional a partir de sua implantação, passando a ser prática comum, fornecendo serviços de qualidade para gestantes e parturiente, evitando mortes maternas, reduzindo taxas de cesáreas e obtendo melhor assistência obstétrica para gestante de baixo e alto risco (Reis et al., 2017). Nesse sentido, de encontro a humanização há o acolhimento, este é oferecido nas instituições públicas por profíssionais da saúde, dentre estes os enfermeiros, muitas vezes, o acolhimento não acontece de forma adequada como regulamenta a Política Nacional de Humanização (PNH), resultando em diversas complicações na assistência à saúde e na relação interpessoal instituição/profissional/paciente por não haver responsabilidade, capacitação e treinamento das equipes em relação a humanização do cuidado a gestante (Grimberg et al., 2015). Desta maneira, a humanização vinculada ao acolhimento requer que os colaboradores da assistência à saúde obstétrica apropriem-se do papel de personagens humanizados, seja no cuidado ou na orientação em saúde, deste modo, esta pesquisa teve por objetivo investigar a compreensão de gestantes e puérperas sobre o Programa de Humanização no Pré-Natal e Nascimento (PHPN) demonstrando que o nível de entendimento pode ser uma premissa no cumprimento de seus direitos a assistência humanizada.

\section{MÉTODOS}

Trata-se de um estudo exploratório-descritivo, com abordagem qualitativa de dados (Dyniewicz, 2009), realizado em uma maternidade pública, localizada no município de Caxias, estado do Maranhão, Brasil. A Maternidade Carmosina Coutinho é uma referência para os atendimentos obstétricos de Caxias e das circunvizinhas. Foram incluídas no estudo gestantes e puérperas maiores de 18 anos, sob internação na maternidade, que concordassem em assinar do Termo de Consentimen to Livre e Esclarecido (TCLE). Foram excluídas pacientes com distúrbios mentais e cognitivos que impedissem a compreensão da entrevista. A amostra final contou com a participação de 15 mulheres, dentre elas, gestantes e puérperas, a coleta dos dados ocorreu no período de março a abril de 2018, a partir de uma entrevista semiestruturada com perguntas abertas acerca da compreensão de gestantes e puérperas sobre o Programa de Humanização no Pré-Natal e Nascimento (PHPN), bem como seus direitos estabelecidos pelo programa. Durante a coleta dos dados, cada participante esteve à vontade para expressar suas opiniões, o levantamento e análise dos dados ocorreu a partir da técnica de observação estruturada e análise de conteúdo de Bardin (2011), na análise da temática, o núcleo de sentido foi identificado possibilitando a exposição das principais expressões revelando as mais importantes informações exteriorizadas pelo sujeito de estudo.

Os dados expostos foram descritos no texto segundo a respectiva paciente investigada: GP1 (Gestante ou Puérpera 1), GP2 (Gestante ou Puérpera 2), GP3 (Gestante ou Puérpera 3) e assim sucessivamente, resguardando a identidade de cada participante. A pesquisa foi aprovada pelo Comitê de Ética em Pesquisa (CEP) da Faculdade de Ciências e Tecnologia do Maranhão - UniFacema com o CAAE de número 83827718.2.0000.8007, comprovante 014924/2018. Os pesquisadores responsáveis comprometeram-se com as normas preconizadas pela Resolução CNS nº 466/12 que trata dos aspectos éticos e legais de pesquisas envolvendo seres humanos (Brasil, 2012).

\section{RESULTADOS}

A ausência de educação e informação em saúde, que se inicia na execução do pré-natal, influencia diretamente no nível de compreensão das gestantes e puérperas quanto aos programas de saúde pública a que tem diretos (Guimarães et al., 2020). Os dados analisados foram obtidos a partir das entrevistas com 15 pacientes sob internação em uma maternidade pública. Quando questionadas sobre seu conhecimento em relação ao PHPN, observou-se os respectivos relatos:

Não, não ouvir falar nesse programa não. Onde eu moro somos tratados super mal pelo povo da saúde, só fala quando a gente pergunta alguma coisa e ainda é com ignorância (GP1).

Não conheço o programa, eles não falaram esse assunto não, nem lá no posto onde fiz meu pré-natal e nem aqui na maternidade (GP2).

Não conheço o programa, sei que temos benefícios mais não fui orientada (GP3).

Não conheço o programa, só ouvir falar depois que cheguei aqui na maternidade, ainda falaram por alto eu não entendi muito bem (GP4).

Não sei desse programa, só conheço mesmo o pré-natal que é obrigatório fazer por causa da criança (GP5).

Nunca ouvir falar nesse tipo de pré-natal e nascimento humanizado, o que eu sei é esse que faz exames e entrega no posto para saber o resultado de como o bebe está (GP6).

Não sei desse programa, só fui informada da importância de fazer o pré-natal e a imunização (GP7).

Não conheço o programa, mas o pré-natal humanizado eu sei que é o acompanhamento que temos que ter quando estamos gestantes, para ver se o bebê está saudável, se estamos tomando os medicamentos certos para não ter problema na hora de parir (GP8).

Sim conheço o programa de pré-natal e parto humanizado, só que eu não sei explicar asações especificas desse programa (GP9).

Não, só sei do pré-natal (GP10; GP11; GP14). Não ouvi falar desses programas, onde eu moro os enfermeiros fazem mal o pré-natal (GP12; GP13).

Não, nunca me falaram de pré-natal, nascimento ou parto humanizado durante as consultas, só escutei falar em parto humanizado hoje na maternidade. Sei que temos direitos, só que não servem muito porque não respeitam (GP15).

Dentre as quinze gestantes e puérperas, apenas uma afirmou conhecer o PHPN, evidenciando o déficit na orientação em saúde das gestantes durante a execução do pré-natal da Unidade Básica de Saúde (UBS). Os discursos das amostras pesquisas demonstram que os profissionais de enfermagem que realizam os pré-natais estão alheios as gestantes, não cumprindo adequadamente com seu dever de orientar e informar sobre seus direitos frente ao programa, sanando dúvidas que poderiam lhes assegurar uma assistência humanizada e acolhedora. As falas transcritas revelam, o desconhecimento das gestantes em relação ao programa e os diretos estabelecidos pelo PHPN voltados a assistência da gestante e puérperas, deste modo, a ausência do conhecimento advém da falta de orientações que deveriam ser 
realizadas pelo enfermeiro da Atenção Básica e responsável pelo prénatal na UBS ou na maternidade e serviços secundários de assistência, onde não ocorre informação e orientação por parte dos profissionais de saúde.

\section{DISCUSSÃO}

O profissional enfermeiro que faz o acompanhamento pré-natal na UBS e não informa a gestante sobre o PHPN e seus direitos demonstra falta de preparo em relação as ações de apoio profíssional, estímulo e motivação na disseminação de conhecimento e aplicabilidade da humanização e direitos da gestante e puérpera nas unidades de saúde e maternidades públicas (Reis et al., 2017). O PHPN estabelece os direitos a assistência de qualidade na gestação parto e puerpério com o sentido de reduzir a morbimortalidade materna-infantil, priorizando a humanização da assistência obstétrica e neonatal, sob a perspectiva da ética profissional e acolhimento em detrimento de práticas que não gerem riscos, comprometendo-se com o planejamento e implementação de ações efetivadas beneficiando sempre o binômio mãe e filho (Melo et al., 2016). O enfermeiro deve orientar e esclarecer a gestante quanto aos seus direitos, programas e políticas de saúde públicas a que tem direito, o que pode facilitar o processo do pré-natal ao pós-parto. As gestantes devem estar conscientes dos seus direitos e programas voltados a sua assistência, a informação e conscientização pode influenciar no cuidado com a própria saúde, prevenindo intercorrências e complicações durante o período gestacional (Rocha; Andrade, 2017; Sales et al., 2016). Na rede pública, atenção pré-natal, é iniciada preferivelmente por um profissional enfermeiro, seguindo para consulta médica, a consulta é um contato que requer a prática de acolhimento tanto para gestante como para seu acompanhante, onde deve haver ações que sejam acolhedoras e esclarecedoras, estimulando a mulher gestante ao desejo de voltar as consultas, conhecer e aceitar aos programas oferecidos pelo SUS (Brasil, 2014). O acompanhamento pré-natal considera como um dos critérios de qualidade o acesso a saúde, o número de consultas, idade gestacional de início de acompanhamento e a adequação dos serviços prestados à gestante. A assistência de qualidade favorece a redução dos índices de mortalidade materna e perinatal, considerando que a disseminação de informação sobre programas de saúde, direitos e orientação de saúde são fundamentais para minimizar estes índices (Polgliane et al., 2014). Segundo Martins, Mattos e Santos (2016), as mulheres devem ter acesso à informação e educação em saúde para compreender melhor o que estão vivenciando no momento e, assim, usar de seus direitos participando de forma efetiva na tomada de decisões em relação ao seu processo parturitivo. Em concordância com esta assertiva, Contreras e Guaymas (2017) ressaltam que a humanização é o fio condutor para ações em saúde eficientes, no entanto, percebe-se que a humanização ainda não é bem esclarecida durante o pré-natal, tarefa que deve ser realizada pelo enfermeiro responsável pelo acompanhamento da gestante.

Para que sempre haja esclarecimentos entre as gestantes, o PHPN visa acolher a mulher neste momento tão especial da gestação, observando, orientando e acompanhando todas as modificações físicas e emocionais da gestante. O pré-natal é onde se inicia o acompanhamento com um conjunto de ações que antecedem o parto e tem como objetivo primordial atender as necessidades da gestante por meio da promoção, prevenção e cuidados. A assistência tem como objetivo o acolhimento da gestante desde o diagnóstico da gestação até as consultas puerperais da mulher e do RN (Rocha; Andrade, 2017). Para que isso ocorra, o acolhimento, seguido da humanização, dever conter escuta sensível e responsabilização diante das especificidades das demandas, valorizando o contexto em que estas são geradas. É fundamental estar atento ao contexto social e familiar em que a gestante está inserida, uma vez que isso pode gerar influências positivas e negativas (Camillo et al., 2016). Deve-se compreender que o acolhimento é uma tecnologia que viabiliza o direito ao acesso e deve ser exercido obrigatoriamente por todas as equipes no atendimento das necessidades singulares da gestante. $\mathrm{O}$ acolhimento é uma conduta plena de ética, respeitando e informando a gestante em todos os seus direitos e orientando-a de forma fiel quanto a Política Nacional de Humanização do SUS (Sales et al., 2016). Conhecer sobre os programas do pré-natal pode contribuído de forma notável para a saúde pública, diminuindo o risco de complicações tanto no pré-natal quanto no pós-parto, através de consultas periódicas com enfermeiros. As consultas de enfermagem são baseadas nos conceitos de prevenção, promoção e vigilância da saúde, que visam garantir o bem-estar e melhorar a qualidade de vida para as gestantes (Rocha; Andrade, 2017). No tocante da humanização e acolhimento no parto, e especialmente ao parto advindo de uma gestação classificada como de alto risco, as maternidades e os profissionais devem estar estruturados adequadamente para atender as necessidades da mulher e do bebê. Espera-se que neste espaço de cuidados, enfermeiros, médicos e demais profissionais movimentem-se em torno do atendimento obstétrico e neonatal de forma humanizada e acolhedora, tratando a gestante de modo digno a assistindo com qualidade (Melo et al., 2016). Em relação aos direitos, além dos direitos sociais de cidadã, a mulher tem direito a assistência integral em toda a gestação, parto, puerpério e acompanhamento do bebê até os 2 anos de idade. Durante o trabalho de parto, a gestante tem direito a um acompanhante de sua escolha, isso por que, vivenciar o trabalho de parto torna-se mais difícil se a mulher estiver sozinha, sem um acompanhante ou familiar de sua escolha, uma vez que surgem sensações de angústia, ansiedade e medo (Correia et al., 2017). É relevante saber que o enfermeiro deve, por meio de informações a respeito de gestação, parto, necessidade de mudanças de hábito, esclarecer dúvidas, como também explicar a importância da realização do pré-natal e das visitas mensais à unidade de saúde. $\mathrm{O}$ acolhimento, cuidado e assistência de enfermagem a gestante não pode se limitar a receber a gestante na atenção primária, trata-se de implantar uma assistência que tem como base a informação, conhecimento e preparo da gestante (Silva; Andrade; Bosi, 2014). Entretanto, a falta de conhecimentos da equipe de enfermagem acerca da prevenção de complicações, manejo para a promoção da saúde, orientações de cuidados e direitos, interfere diretamente no processo de acolhimento. Nesse sentido, as constantes atualizações e capacitações devem fazer parte do programa institucional e de autodesenvolvimento do enfermeiro em busca de uma melhor qualidade assistencial (Moura et al., 2014). Enfim, o enfermeiro nesse processo deve promover um enfoque com maiores cuidados preventivos e ações educativas para que assim consiga melhorar essas assistências prestadas. Logo, quando o profissional se reconhece como sujeito transformador com ações efetivas na assistência e cria vínculo de confiança com essas gestantes, o cuidado passa a ser transformador, mesmo que o serviço seja provido de obstáculos como falta de material, infraestrutura insatisfatória ou ainda recursos humanos insuficientes (Camilloet al., 2016).

\section{CONCLUSÃO}

Diante do exposto, percebe-se a fragilidade da enfermagem no que tange a orientação e conscientização das gestantes quanto o Programa de Humanização no Pré-Natal e Nascimento no âmbito do Sistema Único de Saúde, observa-se uma lacuna na qualidade da prestação dos serviços de acompanhamento pré-natal, principalmente no sentido de garantir informações sobre a Humanização no Pré-Natal e Nascimento. Durante o acompanhamento das gestantes, o enfermeiro da atenção básica, principal responsável pelo acompanhamento do pré-natal, deve instruir gestantes e seus acompanhantes quanto a importância da Humanização no Pré-Natal e Nascimento, bem como, seus direitos frente os princípios de diretrizes do programa. É fundamental a colaboração dos enfermeiros na promoção da saúde, conduzir as gestantes para que compreendam adequadamente seus direitos ao pré-natal adequado, acesso aos serviços da maternidade de referência para seu atendimento, assistência segura, integra e humanizada no parto e puerpério, bem como, assistência ao recémnascido. Conhecer o Programa de Humanização no Pré-Natal e Nascimento traz autonomia para a gestante e participação efetiva no seu processo gestacional e parturitivo, uma vez que, compreender sobre o programa pode ser uma premissa para exigir o cumprimento de seus direitos a assistência humanizada. 


\section{REFERÊNCIAS}

Bardin L. (2011). Análise de conteúdo. Ed. 70. São Paulo.

Brasil. (2002) Ministério da Saúde. Secretaria de Atenção à Saúde. Departamento de Ações Programáticas Estratégicas. Programa Humanização do parto: Humanização do pré-natal e nascimento. Brasília.

Brasil. (2012). Ministério da Saúde. Conselho Nacional de Saúde. Resolução $n^{\circ} 466$, de 12 de dezembro de 2012. Dispõe sobre diretrizes e normas regulamentadoras de pesquisas envolvendo seres humanos. Brasília (p.64).

Brasil. (2012). Ministério da Saúde. Secretaria de Atenção à Saúde. Departamento de Atenção Básica. Atenção ao pré-natal de baixo risco. Cadernos de Atenção Básica, $\mathrm{n}^{\circ} 32$. Editora do Ministério da Saúde (p. 318).

BRASIL. (2014). Ministério da Saúde. Secretaria de Atenção à Saúde. Departamento de Ações Programáticas Estratégicas. Manual de acolhimento e classificação de risco em obstetrícia. Brasília.

Camillo, B. S., Nietsche, E. A., Salbego, C., Cassenote, L. G., Dal Osto, D. S.\& Böck, A. (2016). Health educationactions in primaryattentiontopregnantand puerperal women: integrative review. Rev. enferm. UFPE online, 10(6), 4894-4901.

Contreras, M. \&Guaymas, M. (2017). Nursingcare for a humanidezchildbirth. Notas enferm. (Córdoba), 17(29), 9-15.

Correia, S. R. Silva, J. M. de O. e, Santos, A. A..P. dos, Comassetto, I., Lima, G. K. S. de\& Ferreira, D. C. da S. (2017). Nursingcaretoadolescentwoman in labor in the light of Wanda Horta theory. Rev. pesqui. cuid. fundam.,9(3), 857-866.

Diniz, C. S. G., d'Orsi, E., Domingues, R. M. S.M., Torres, J. A., Dias, M. A. B.,Schneck, C. A.,Lansky, S., Teixeira, N. Z. F., Rance, S. \&Sandall, J. (2014). Implementação da presença de acompanhantes durante a internação hospitalar para parto: dados do inquérito nacionalNascer no Brasil. Cadernos de Saúde Pública, 30(Supl. 1), S140-S153.

Dodou, H. D., Rodrigues, D. P. \& Oriá, M. O. B. (2017). O cuidado à mulher no contexto da maternidade: caminhos e desafios para a humanização. J.Rev. Fundam. Care. Online, 9(1), 222-230.

Dyniewicz, A. M. (2009). Metodologia da pesquisa em saúde para iniciantes. 2. ed. São Caetano do Sul - SP: Difusora editora.
Guimarães, Q. V., Coêlho, L. P. I., Santos, M. B. L. dos, Costa, A. C. M. da, Pereira, B. M. \& Silva, J. T. P. (2020). Vivência de puérperas diante da assistência obstétrica no processo parturitivo. SANARE, 19(1), 48-57.

Martins, C. A.; Mattos, D. V.; Santos, H. F. L. (2016). Woman'sautonomy in thechildbirthprocess. Rev. enferm. UFPE online, 10(12) 4509-4516.

Melo, M. N., Amorim, T. V., Salimena, A. M. de O., Melo, M. C. S. C.de\&Souza, Í. E. de O. (2016). Careofwomenthatexperienced a high riskpregnancy: contributionstonursing. Rev. enferm. UFPE, 10(11), 3911-3917.

Moura, M. A. V., Fernandes, G. e-S., Santos, C.\&Mendes, V. de A. S. (2014). A qualidade de assistência de enfermagem no período pré-natal na perspectiva da mulher gestante. Aquichan, 14(2), 196-206.

Nóbrega, J. A. dos S., Assis, C. M. L., Guimarães, D. A., Farias, E. de S., \&Grimberg, S. K. C. R. (2016). Barriers in receptivenessby nurses of a public hospital. Revista Brasileira De Ciências Da Saúde, 19(4), 299-306.

Polgliane, R. B. S., Leal, M. do C., Amorim, M. H. C., Zandonade, E., \& Santos Neto, E. T. dos. (2014). Adequação do processo de assistência pré-natal segundo critérios do Programa de Humanização do Pré-natal e Nascimento e da Organização Mundial de Saúde. Ciência \& Saúde Coletiva, 19(7), 1999-2010.

Reis, T. L. da R. dos, Padoin, S. M. de M., Toebe, T. R. P., Paula, C. C. de, \& Quadros, J. S. de. (2017). Women'sautonomy in theprocessoflabourandchildbirth: integrativeliterature review. Revista Gaúcha de Enfermagem, 38(1), e64677.

Rocha, A. C. \& Andrade, G. S. (2017). Nursing team attention during prenatal: perception of pregnant women assisted in basic network at Itapuranga-GO in different social contexts. Revista Enfermagem Contemporânea, 6(1), 30-41.

Sales, C. de O. C. B., Lopes, M. F., Silva Júnior, G. B. da\& Frota, M. A. (2016).UnderstandingandrecognitionoftheStork Network for Family Health Strategy. Nursing(São Paulo), 17(222), 12711275.

Silva, M. Z. N., Andrade, A. B. \& Bosi, M. L. M. (2014). Access and user embracement in prenatal care through the experiences of pregnant women in Primary Care. Saúde debate, 38(103), 805816. 